\title{
Cambios en la Televisión Pública argentina
}

\section{Changes in argentine Public Television}

\section{Yamila Heram}

Universidad de Buenos Aires, Argentina

$$
\left(\begin{array}{l}
\text { Fecha de recepción: } 28 \text { de noviembre de } 2019 \\
\text { Fecha de aprobación: } 11 \text { de mayo de } 2020
\end{array}\right)
$$

\section{NOTAS BIOGRÁFICAS}

Yamila Heram es doctora en Ciencias Sociales por la Universidad de Buenos Aires (UBA), Magíster en Comunicación y Cultura y Licenciada en Ciencias de la Comunicación (UBA). Investigadora Adjunta del Consejo Nacional de Investigaciones Científicas y Técnicas (CONICET). Docente en la materia "Teorías y Prácticas de la Comunicación II" y del Seminario "Televisión y Crítica de Medios" en la Facultad de Ciencias Sociales de la Universidad de Buenos Aires.

Contacto: yaheram@yahoo.com.ar

\section{Resumen}

Este trabajo parte del interés por reflexionar en torno a la Televisión Pública argentina, entendiendo que es el medio que puede, debe y tiene los recursos necesarios para proponer otro tipo de programación que sepa reunir aspectos vinculados a lo local y lo regional, que ofrezca nuevas propuestas de ficción, que incluya una mayor diversidad de representaciones, que se preocupe por obtener una audiencia más amplia, que promueva debates y una pluralidad informativa. En objetivo es realizar un análisis comparativo de la grilla de programación durante la gestión del macrismo para observar tendencias, cambios y continuidades en la propuesta de la emisora en dos períodos: marzo y abril de 2016 (al inicio de la gestión) y un segundo período que es marzo y abril de 2019. A los fines del análisis se recurre a una amplia literatura proveniente de la economía política de la comunicación que permite contextualizar y comprender el estado actual de la emisora. Se toma una muestra acotada que funciona como "caso testigo" y permite comparar y analizar la programación. Se establecen diversos ejes de análisis: promedio semanal de tiempo de emisión por contenidos referenciales, ficcionales e híbridos; géneros que predominan en cada uno de ellos; programación del prime time, y país de origen del producto transmitido. La información obtenida es abordada desde un análisis cualitativo para identificar invariantes, constantes que solo son posible en un análisis cultural de la oferta televisiva. Entre las principales conclusiones nos interesa destacar una palabra -tensión- que sintetiza el estado de situación de la emisora, hacemos referencia a: géneros referenciales diversos en el horario del prime time y un nivel de audiencia de los más bajos; mayor audiencia durante la gestión anterior y una pluralidad informativa cuestionada; mayor emisión de productos locales y disminución de inversión en los medios públicos.

\section{Abstract}

This work starts from the interest in reflecting on argentine Public Television, understanding that it is the medium that can, should and has the necessary resources to propose another type of programming that knows how to bring together aspects related to the local and the regional, that offers new fictional proposals that include a greater diversity of representations, that are concerned with obtaining a wider audience, that promote debates and a plurality of information. The objective 
is to carry out a comparative analysis of the programming grid during the management of the macrismo to observe trends, changes and continuities in the station's proposal in two periods: March and April 2016 (at the beginning of the management) and a second period which is March and April 2019. For the purposes of the analysis, a broad literature from the political economy of communication is used, which allows contextualizing and understanding the current state of the station. A bounded sample is taken that functions as a "control case" and allows the programming to be compared and analyzed. Various analysis axes are established: weekly average of broadcasting time by referential, functional and hybrid content; genres that predominate in each of them; prime time programming, and country of origin of the transmitted product. The information obtained is approached from a qualitative analysis to identify invariants, constants that are only possible in a cultural analysis of the television offer. Among the main conclusions we are interested in highlighting a word -voltage- that synthesizes the state of the station, we refer to: different referential genres in prime time and a low audience level; greater audience during the previous administration and a questioned plurality of information; higher emission of local products and decreased investment in public media.

\section{Palabras clave}

Televisión Pública, grilla de programación, medios públicos, Argentina, macrismo.

\section{Keywords}

Public televisión, TV grid, public mass media, Argentine, macrismo.

\section{Sumario}

1. Introducción

2. Metodología y estado de situación de la Televisión Pública

3. Resultados

3.1. Contenidos referenciales, ficcionales e híbridos

\subsection{Géneros televisivos}

\subsection{Prime Time}

\subsection{País de origen}

4. Conclusiones

\section{Summary}

1. Introduction

2. Methodology and status of Public Television

3.1. Referential fictional and hybrid contents

\subsection{TV genres}

\subsection{Prime Time}

\subsection{Country of origen}

4. Conclusions 


\section{INTRODUCCIÓN}

La temática de los medios públicos ha sido ampliamente trabajada por parte del campo comunicacional latinoamericano ${ }^{1}$, en el caso de Argentina desde una diversidad de perspectivas y enfoques se ha indagado en torno a las políticas comunicacionales, su contenido, su historia ligada a los intereses gubernamentales y la relación con las audiencias, entre otros aspectos. Este trabajo se enmarca en el interés por reflexionar en torno a la Televisión Pública argentina, en un aspecto no tan desarrollado como es la programación de la emisora. En trabajos anteriores (Heram, 2018; Heram et al, 2017) hemos indagado sobre la programación del canal, advirtiendo ciertas características durante la gestión del macrismo iniciada a principios de 2016 y finalizada del 10 de diciembre de 2019. En este trabajo nos proponemos realizar un análisis comparativo de la grilla de programación durante dicha gestión, para observar las tendencias, cambios y continuidades en la propuesta televisiva de la emisora en dos períodos: el primero durante marzo y abril de 2016 momento de lanzamiento de la programación en los primeros meses de gestión del macrismo y un segundo período correspondiente a la programación de marzo y abril de 2019. Para ello, tomamos una muestra acotada que funciona como "caso testigo" y permite - a partir de los resultados obtenidos previamente (Heram, 2018)comparar el estado actual de la programación.

El interés de indagar en torno a los medios públicos, específicamente en la Televisión Públi$c a$, parte de entender que es el medio que puede, debe y tiene los recursos necesarios para proponer otro tipo de programación que sepa reunir aspectos vinculados a lo local y lo regional, que ofrezca nuevas propuestas de ficción, que incluya una mayor diversidad de representaciones, que se preocupe por obtener una audiencia más amplia, que promueva debates y una pluralidad informativa. En Argentina el sistema de medios está conformado por medios de gestión privada, estatal y sin fines de lucro $^{2}$. La Televisión Pública es un canal de gestión estatal de alcance nacional que forma parte del Sistema de Medios Públicos estatales que integran Radio y Televisión Argentina (RTA). Es conocido y ha sido ampliamente trabajado por diversos autores, la larga tradición de los medios de gestión estatal y su estrecha relación con los intereses gubernamentales (Becerra, 2015; Ortega, 2010; Portales Cifuentes, 2005; Mindez, 2001; entre otros), oficiando históricamente como medio de propaganda de los gobiernos de turno. No es un detalle menor esta ambigüedad entre su tradición gubernamental, su gestión estatal y su marca institucional como televisión pública, o como menciona Becerra "la ausencia de una tradición potente de medios públicos en América Latina" (2013, p. 48). Partimos de entender a la Televisión Pública como un medio que ofrece un servicio público a la ciudadanía ${ }^{3}$, por ende, un análisis comparativo sobre la programación de la emisora permitirá comprender los cambios y continuidades, sus prioridades y ausencias.

\section{METOdOLOGÍA Y ESTADO DE SITUACIÓN DE LA TELEVISIÓN PÚBLICA}

La metodología utilizada para este trabajo está en relación con el objetivo del artículo que es analizar y comparar la grilla de programación de la Televisión Pública en dos períodos de la actual gestión. Este trabajo se enmarca en una investígación mayor ${ }^{4}$ que ha arrojado los primeros resultado en torno a las características de la programación de los canales abiertos (Heram et al, 2017) y de la emisora en particular en 2016 (Heram, 2018). A partir de estos resultados, en los que se tomó como unidad mínima de análisis una muestra acotada que comprendió las últimas dos semanas de marzo y abril de 2016 -estos meses se corresponden con el cambio y paulatina transición de la programación de la nueva gestión ${ }^{5}$. Al presente trabajo se le sumó como unidad mínima de análisis las mismas semanas de la grilla de programación de 2019. La muestra está conformada por dos momentos significativos, el inicio de una nueva propuesta en 2016 y en 2019 la consolidación de la misma. Se opta por tomar una semana completa ya que consideramos que es la unidad mínima de análisis, la grilla de programación se organiza semanalmente -en relación con los tiempos de la cotidianeidad social en términos de Martín Barbero (1987) - y esta investigación es de corte cualitativo (Denzin y Lincoln, 2003). Es decir, se describe la grilla de programación del canal y se resaltan sus características más recurrentes, ello permite identificar invariantes para realizar el análisis cultural de la programación televisiva.

El principal motivo por el cual consideramos relevante realizar este análisis comparativo parte de entender que durante la gestión del macrismo se han suscitado una serie de cambios en torno a los medios públicos que han afectado tanto a nivel presupuestario, así como de producción y difusión. Por solo nombrar algunos de los más sobresalientes, tras la asunción de Mauricio Macri como presidente en diciembre de 2015 se 
realizaron una serie de modificaciones legales. El 4 de enero de 2016 el Decreto de Necesidad y Urgencia (DNU) 267/156 modificó artículos de la Ley de Servicios de Comunicación Audiovisual y creó el Ente Nacional de Comunicaciones (ENaCom $)^{7}$. Explica Becerra (2016) que el DNU apuntó en dos direcciones, por un lado: "toma el control de los órganos de regulación y aplicación de la política de medios y telecomunicaciones y los subordina más al Poder Ejecutivo Nacional” (p. 2) y por el otro:

Modifica la regulación sobre concentración de la propiedad en tv abierta, tv cable y radios, extiende el plazo de explotación de todas las licencias audiovisuales en vigor y habilita el cruce para que grupos audiovisuales puedan brindar servicios de telecomunicaciones y (más tarde) para que los grupos de telefonía puedan ofrecer televisión por cable.

En diciembre de 2015 se creó el Sistema Federal de Medios y Contenidos Públicos (SFMyCP), y se nombra como titular a Hernán Lombardi. Según el informe Media Ownership Monitor (MOM) desarrollado por el diario Tiempo Argentino y Reporteros sin Fronteras, a nivel presupuesto hubo un congelamiento del dinero en los medios públicos, "en 2018 el presupuesto fue igual al del año 2017 con una inflación interanual de más del 40\%" (2019, p. 1026). Esta baja de inversión se volcó en los trabajadores, según este mismo informe en 2017 se despidieron a 160 personas de los canales que formaban parte de los medios públicos: Encuentro, PakaPaka y DeporTV. En la Agencia de noticias Télam se despidieron a 354 trabajadores $^{8}$. También se dejó de emitir Fútbol para todos, es decir se volvió a privatizar la transmisión de Fútbol de Primera División. En cuanto a la situación de la Televisión Pública se realizaron retiros voluntarios y además hubo 11 despidos. Asimismo, según los datos ofrecidos por el informe MOM, la emisora "perdió más 40\% del encendido entre 2015 y 2018" (2019, p. 1027).

Bajo este estado de situación es que el análisis de la grilla de programación permitirá continuar reflexionando en torno al devenir del canal. Realizamos el análisis de las grillas a partir de la propuesta metodológica de Orza (2002), que consiste en un modelo integral para el análisis de la programación televisiva, que permite acceder a una catalogación genérica y caracterización de las unidades discursivas de la grilla.

En primer lugar se realizó el visionado de la programación y la recolección de los datos, para ello se tomó la información brindada por la propia página web del canal y se registró la siguiente información: día, horario, nombre del programa, tipo de discurso, género televisivo y país de origen del producto. Orza (2002) distingue los tipos de discursos en relación con los campos de referencia en que se organizan: externos -discurso referencial-, internos -ficcional- o mixtos -hibridación-. Por referencial hace alusión a aquellos que representan sus contenidos en relación con el campo de referencia externo, se podría ubicar en esta categoría a los programas de noticieros, debate político, documentales, periodístico, transmisión en directo de deporte, cultural, religioso, entre otros. En los contenidos ficcionales se ubican los que representan un campo de referencia imaginario o fantástico aunque tengan aproximación con la realidad externa, por ejemplo los géneros de las telenovelas, series, dibujos animados, películas, unitario, entre otros. Los discursos de hibridación son aquellos que representan sus contenidos combinando las formas de aproximación a lo ficcional y a lo referencial. Algunos de los géneros que pueden ubicarse dentro de este tipo de discurso son: magazine, entretenimiento, programas de espectáculos y reality-show, entre otros.

Una vez obtenida toda la información, la analizamos a partir de los siguientes ejes: promedio semanal de tiempo de emisión por contenidos referenciales, ficcionales, e híbridos; géneros que predominan en cada uno de ellos; programación en el horario de prime time, país de origen del producto transmitido. En primer lugar se sistematizó la información por mes y año y posteriormente se realizó la comparación entre 2016 y 2019 a los fines de observar las continuidades y modificaciones en las tendencias de las grillas. La información obtenida fue abordada desde un análisis cualitativo para identificar invariantes, constantes que sólo son posibles en un análisis cultural de la oferta televisiva. Asimismo, vale aclarar que este trabajo no sólo dialoga con una amplia literatura que desde la economía política de la comunicación ha analizado las políticas comunicacionales en torno a los medios públicos, sino también con una serie de informes desarrollados por diferentes organismos, observatorios e instituciones que aportan y contextualizan diversas aristas en torno a la Televisión Pública.

\section{RESULTADOS}

Analizar la grilla de programación de un canal es preguntarnos por la manera en que la emisora pensó su propuesta televisiva, la cual funciona como una carta de presentación de cara al público que desea llegar. En el caso de la Televisión 
Pública la primera cuestión a mencionar es la clara diferenciación de su propuesta en relación con las emisoras privadas. Por ejemplo, durante los fines de semana ofrece una propuesta diversa en términos de contenidos: programas religiosos que representan a los tres cultos monoteístas: cristianismo, judaísmo e islamismo, siendo el católico el que tiene mayor tiempo de pantalla, también se transmite en vivo el deporte de automovilismo, se ofrecen productos culturales, películas, así como el programa de las Madres de Plaza de Mayo, del Ejército Argentino, entre otros. Sin embargo, durante la semana la programación se suele reiterar de lunes a viernes en el horario que va desde las 6:00 am hasta la medianoche cuestión similar sucede en los medios privados-, y durante la madrugada se repiten programas ya emitidos, variando éstos según los días. En marzo de 2016 se emitieron 53 programas semanales, en abril del mismo año 46. En marzo de 2019, 42 programas y en abril del mismo año 51.

\subsection{CONTENIDOS REFERENCIALES, FICCIONALES E HÍBRIDOS}

Como se mencionó en el apartado anterior y siguiendo a Orza (2002), los discursos se pueden distinguir en relación con los campos de referencia en que se organizan: referenciales, ficcionales e híbridos. Compartimos los gráficos con el porcentaje semanal de tiempo de emisión por tipo de discurso en ambos períodos.

Porcentaje semanal de tiempo de emisión por tipo de discurso

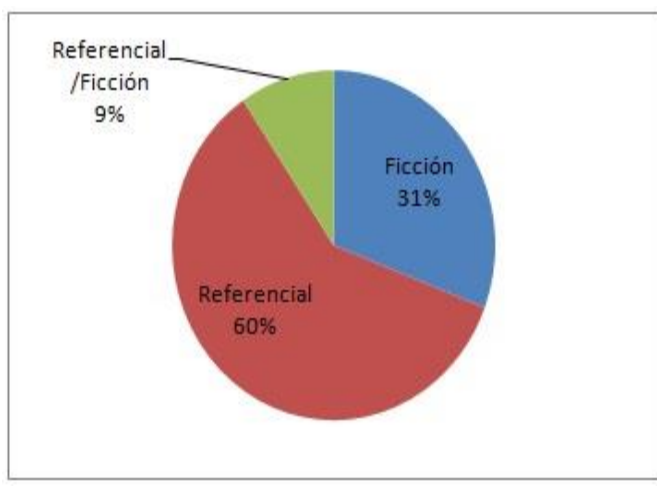

Marzo, del 28/03/16 al 03/04/16

Gráficos de elaboración propia

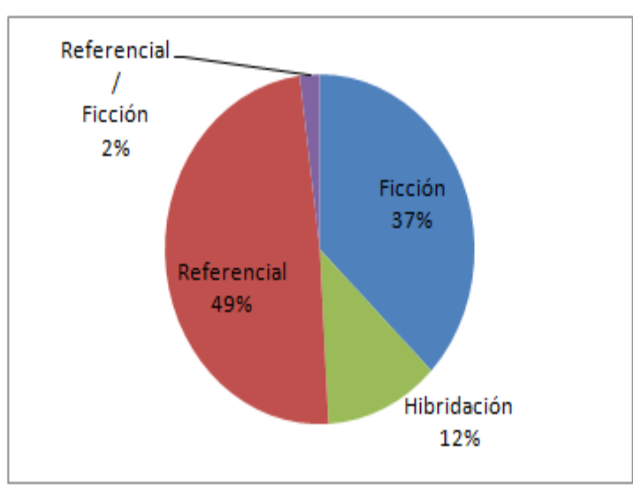

Abril, del 25/04/16 al 01/05/2016

Porcentaje semanal de tiempo de emisión por tipo de discurso

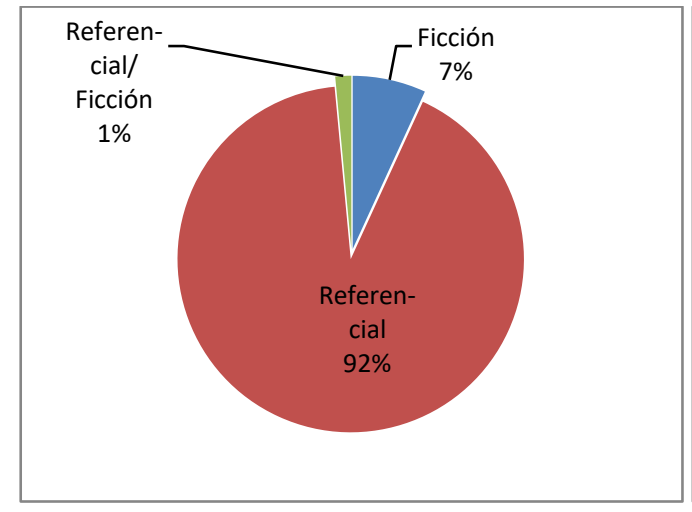

Marzo, del 23/03/19 al 30/03/19

Gráficos de elaboración propia

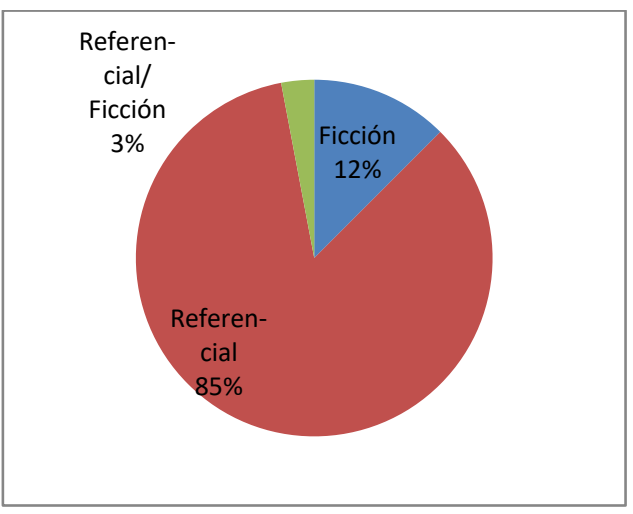

Abril, del 20/04/19 al 27/04/19 
De los gráficos se desprende una variación en los tipos de discurso entre 2016 y 2019, aumentando más de un $30 \%$ el tipo de discurso referencial, disminuyendo lo ficcional un $25 \%$ y lo referencial-ficción un $7 \%$. Otra diferencia es que durante marzo y abril de 2019 no se emitieron contenidos de hibridación, cuestión que en abril de 2016 sí.

Cabe aclarar que por "referencial-ficción" hacemos referencia al bloque que se emite del canal infantil PakaPaka ${ }^{9}$. Ha sido denominado de esta manera porque se incluyen tanto dibujos animados correspondientes al discurso de ficción como documentales que lo ubicamos como referencial. Una cuestión importante a señalar es la disminución en términos porcentuales de los contenidos vinculados con los más pequeños. Creemos que esto se corresponde con la reducción en términos generales de la producción propia de los medios públicos, como se menciona en el informe de Reporteros sin Fronteras "la TVP dejó de producir contenidos en vivo durante los fines de semana, mientras Radio Nacional comenzó a retransmitir la programación de Buenos Aires en las frecuencias de FM de sus repetidoras en todo el país (...). En Encuentro, PakaPaka y DeporTV, las producciones propias se redujeron al mínimo por el recorte en los puestos laborales" (2019, p. 1027). Los contenidos emitidos en la Televisión Pública de la señal Pakapaka son de 1\% y 3\% en marzo y abril de 2019, coincidimos con SánchézCarrer y Médiz-Rojas (2013) y Salviolo (2013) que estos contenidos incentivan un aprendizaje activo e interactivo, los niños son pensados no como mero receptores. El descenso en cantidad de horas de emisión de estos contenidos se aleja de una de las funciones de los medios públicos, en términos de promover propuestas educativas y pedagógicas para diferentes rangos etarios.

Algunos datos que aporta Smerling (2018) sobre la disminución de horas de programación que sufrió PakaPaka -cuestión que se ve reflejada en la TVP como ya mencionamos- "de 87,60 horas de producción propias (...), se pasó a menos de la mitad en 2017: fueron, en total, ocho series, cinco micros y una app, unas 37,55 horas. En 2018, la cifra (aunque parcial porque es del mes de agosto) es de seis producciones propias, con un total de 22.75 horas" (s/p). En términos presupuestarios PakaPaka "pasó de 101.626.748 pesos en 2016 a 36.499.652 en 2017 (casi dos tercios menos), y en 2018 alcanzó su mínimo histórico: solo 6.583.231 pesos" (s/p).
Estos datos ayudan a contextualizar y comprender el descenso que sufrió la señal infantil en la propuesta de la Televisión Pública.

Otro dato sobresaliente es la disminución, en 2019 , de un $25 \%$ en tiempo de emisión de los contenidos ficcionales, ello se debe a la ausencia de fomento a la ficción por falta de inversión (Monje, Zanotti y Rivero, 2017). Esta tendencia si bien comenzó con la nueva gestión fue haciéndose visible paulatinamente. Como mencionan Aprea, Kirchheimer y Rivero (2017):

La TV Pública, con el nuevo gobierno, reorienta la política en relación con el fomento de la producción de ficciones independientes. Durante la primera mitad del año [2016], se paralizó la producción debido a la revisión de las inversiones realizadas hasta el momento. Cuando se reanudó la actividad se priorizaron los proyectos ligados a las perspectivas más comerciales (p. 84).

Además, es importante señalar que muchas de las series que se estrenaron durante 2016 provenían de los concursos de ficción que se habían realizado durante la gestión anterior. Asimismo, de lo que va de 2016 a 2019 es notoria la disminución de horas de pantalla que ocupa la ficción en la Televisión Pública.

Por último, mencionar una ausencia significativa en la propuesta de la emisora, hacemos referencia a los contenidos de hibridación que sólo durante abril de 2016 ocupan una cuota de pantalla menor (12\%). Ello se debe a que la programación de la Televisión Pública se basa en una diferenciación en términos de contenidos con los canales privados -sobre ello se ampliará en el siguiente apartado - que ofrecen una amplia propuesta generalistas basada en el panelismo televisivo (Heram, 2018), a través de diferentes géneros correspondientes a los contenidos de hibridación -en especial Canal 9 y América TVtales como el magazine, los programas de espectáculos y de entretenimiento, entre otros.

\subsection{GÉNEROS TELEVISIVOS}

En el siguiente apartado nos detenemos en los géneros televisivos que predominan por tipo de discurso. Compartimos los siguientes gráficos de ambos períodos por promedio de tiempo de emisión del discurso referencial. 


\section{ARTÍCULOS}

Porcentaje de tiempo de emisión por género del discurso referencial

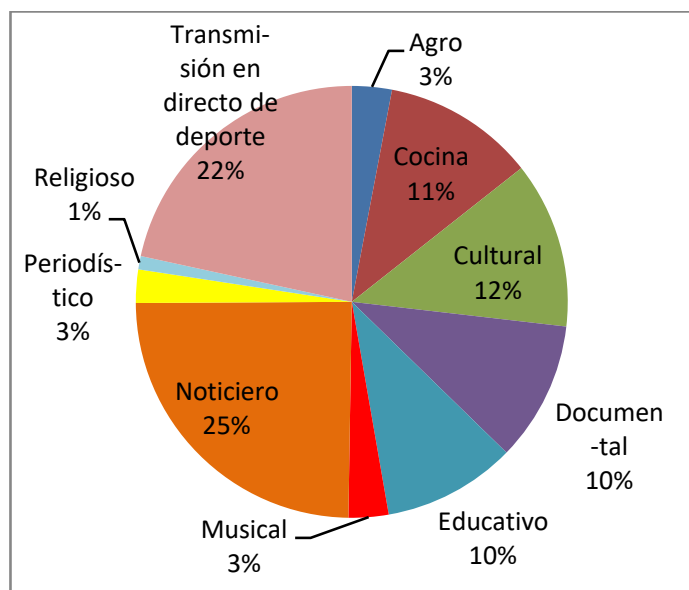

Marzo, del 28/03/16 al 03/04/16

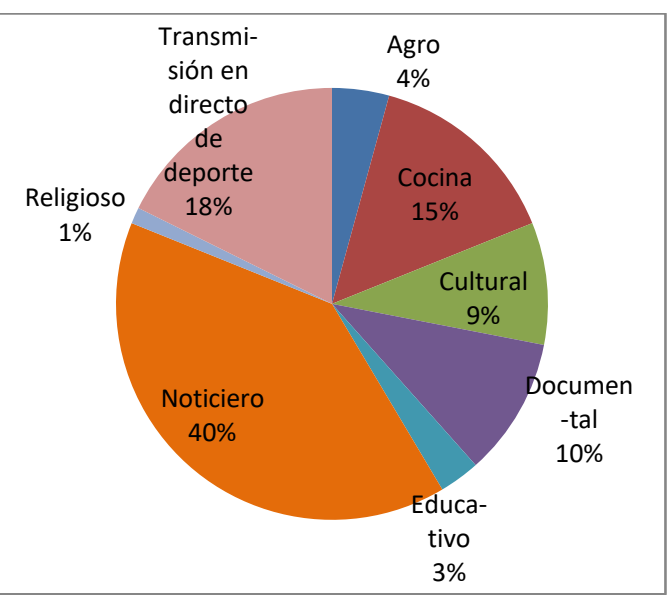

Abril, del 25/04/16 al 01/05/2016

Gráficos de elaboración propia

Porcentaje de tiempo de emisión por género del discurso referencial

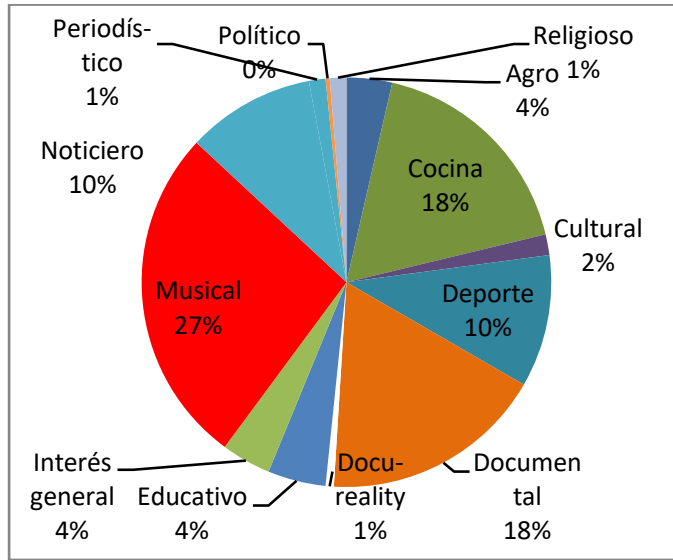

Marzo, del 23/03/19 al 30/03/19

Gráficos de elaboración propia

Como se mencionó en el apartado anterior el tipo de discurso que predomina en la Televisión Pública es el referencial. La programación está compuesta por una amplia variedad de géneros televisivos vinculados con lo educativo, documental, religioso, cultural, cocina, entre tantos otros. En consonancia con la gran cantidad de horas semanales que ocupa este tipo de discurso y su incremento de 2016 a 2019, es que también se han incorporado nuevos géneros televisivos. En marzo de 2016 se emitieron 10 géneros y en el mismo mes de 2019 12, es decir dos más correspondientes al Docu-reality e Interés General. En abril de 2016 se emitieron 8 y en el mismo mes de 2019,12 , se sumaron 4 géneros nuevos, ellos

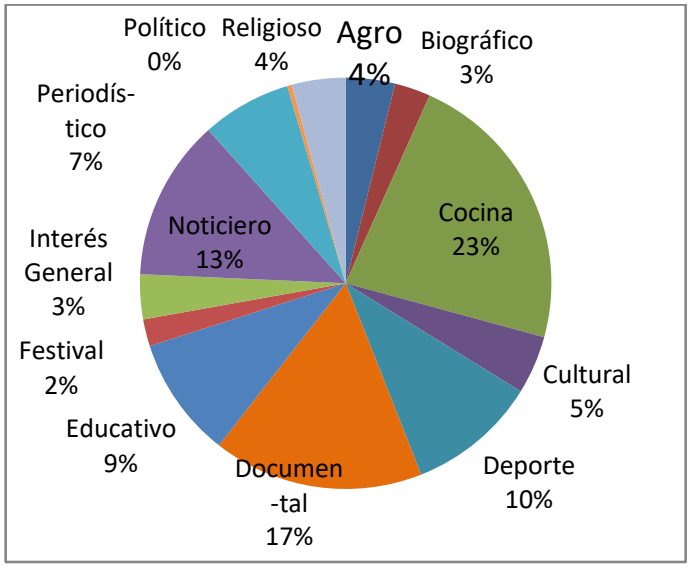

Abril, del 20/04/19 al 27/04/19

son: Biográfico, Festival, Interés General y Periodístico.

La variedad de géneros en lo que respecta al discurso referencial es una de las características diferenciadoras de la emisora para con los medios privados. Sin embargo, una de las tensiones en torno a los medios públicos es si estos deben ofrecer contenidos que los privados no realizan por motivos de audiencia, rentabilidad y/o costos (Martín Barbero, 2005; Rincón, 2005). Por ejemplo, algunos autores focalizan en la cuestión de lo educativo y cultural. Martín Barbero (2005) menciona que los medios públicos deben brindar contenidos culturales y de calidad, Ricaurte (2009) propone ofrecer contenidos con compromiso en la educación y el arte. Ambos autores plantean dar espacio al género educativo que 
promueva valores ciudadanos como una de las metas de los medios públicos. Si bien, el porcentaje de tiempo de emisión de los programas culturales descendió de 2016 a 2019, otros se han mantenido como el educativo, sin embargo la mayor diferencia es que en 2016 este tipo de géneros eran transmitidos en los horarios de menor encendido y en 2019 han pasado al horario del prime time. Ahora bien, este tipo de género que sería uno de los que mejor representa al tipo de programación que, una parte del campo académico, le pide a los medios públicos, entra en tensión con el llegar a un público cada vez más amplio, es decir, la preocupación por las audiencias (Rincón, 2005).

Es cierto que el nivel de audiencia de Televisión Pública ha sido minoritario en comparación con el resto de los canales de aire, pero en los últimos años ha descendido aún más. Tal como se menciona en el informe de Reporteros sin Fronteras: "los niveles de audiencia, que mostraron una fuerte retracción en todos los medios de la televisión abierta argentina en los últimos años, cayeron un 50\% entre 2015 y 2017 en el canal estatal, el de mayor retracción" (2019, p.
65). También cabe mencionar que, paradójicamente durante la gestión anterior, los mayores índices de audiencia se correspondían con algunos programas que ponían en cuestión uno de los aspectos en que la mayoría de los autores coinciden: los medios públicos deben brindar pluralidad informativa ${ }^{10}$. Del monitoreo de los noticieros de los canales de aire que realizó la Defensoría del Público de Servicios de Comunicación Audiovisual en 2014 se obtienen algunos datos que son útiles para comprender las tendencias políticas que preponderaron en cada emisora. Al respecto Becerra (2015) menciona:

Si bien no existe consenso sobre el significado del pluralismo político, y distintas regulaciones en otros países ensayan variantes sobre cómo alcanzar una información plural política, lo cierto es que la predominancia de una fuerza política, es decir, la exhibición de una perspectiva en detrimento de otras opiniones, reducen la diversidad de puntos de vista (p. 105).

Becerra (2015) grafica esta tendencia de las emisoras en un cuadro en el que mide el tiempo de palabra de las fuerzas parlamentarias en los noticieros de la televisión abierta.

Tiempo de palabra de las fuerzas parlamentarias en los noticieros

\begin{tabular}{|l|l|l|l|}
\hline Medio & $\begin{array}{l}\text { Frente para la } \\
\text { Victoria }\end{array}$ & Otros & Observaciones \\
\hline Canal 7 & 67.6 & 31.4 & $\begin{array}{l}\text { La segunda fuerza en tiempo asignado detrás del } \\
\text { FPV es UNEN, con 5,1\%. }\end{array}$ \\
\hline Canal 13 & 30.9 & 67 & $\begin{array}{l}\text { EL FVP duplica al segundo más difundido (UCR), al } \\
\text { que se le dedicó el 18,2\%. }\end{array}$ \\
\hline $\begin{array}{l}\text { Telefe } \\
\text { (Canal 11) }\end{array}$ & 45.2 & 54.8 & $\begin{array}{l}\text { UNEN fue la segunda fuerza, detrás del FPV, con el } \\
35 \% .\end{array}$ \\
\hline $\begin{array}{l}\text { Canal 9 } \\
\text { La segunda fuerza más difundida fue el Frente } \\
\text { Renovador, con el 20\% (un tercio del FPV). }\end{array}$ \\
\hline $\begin{array}{l}\text { AméricaTV } \\
\text { Canal 2) }\end{array}$ & 61.5 & 35.6 & Le asignó un 51,4\% del tiempo al Frente Renovador. \\
\hline
\end{tabular}

Cuadro elaborado por Becerra (2015)

Encontramos, entonces, una suerte de tensión entre mayor audiencia y menor pluralismo; si bien históricamente la emisora ha tenido bajos niveles de audiencia, en los últimos años esta tendencia se ha acrecentado (Becerra y Schejtman, 2018). Según los datos de KANTAR/IBOPE Media en 2015 el promedio anual de la emisora fue de 2.8 y en abril de 2019 de 0.8 .

También cabe mencionar que uno de los cambios más notorios, en cuanto a los géneros, es el descenso en más de un $20 \%$ en tiempo de emi- sión de los noticieros ${ }^{11}$, que dejaron de emitirse los fines de semanas y además "en materia periodística el canal prescindió de los columnistas y analistas en todos los noticieros, quienes se encargaban de aportar contexto y análisis para una comprensión más profunda de los temas" (Monje, Zanotti y Rivero, 2017, p. 165). Como menciona Becerra "los medios estatales ya no son tan intemperantes, pero dejaron de interesar a un sector importante de la sociedad y cedieron terreno en beneficio de los emisores privados comerciales" (2016, s/p). Con respecto a los noti- 
cieros, un dato interesante que aporta el informe de 2017 sobre el monitoreo de los programas noticiosos de los canales de aire de la Ciudad Autónoma de Buenos Aires realizado por la Defensoría del Público de Servicios de Comunicación Audiovisual, es que la Televisión Pública fue el canal que menos fuentes utilizó al momento de emitir las noticias. Si bien el informe es sobre 2017 es un dato a considerar a la hora de refle- xionar sobre la mencionada pluralidad informativa.

En cuanto a los géneros por discurso de ficción, como se mencionó en el apartado anterior, de 2016 a 2019 este descendió en tiempo de emisión un 25\%, y ello se ve reflejado en los géneros que se emiten. Compartimos los gráficos que así lo ilustran.

Porcentaje de tiempo de emisión por género del discurso ficcional

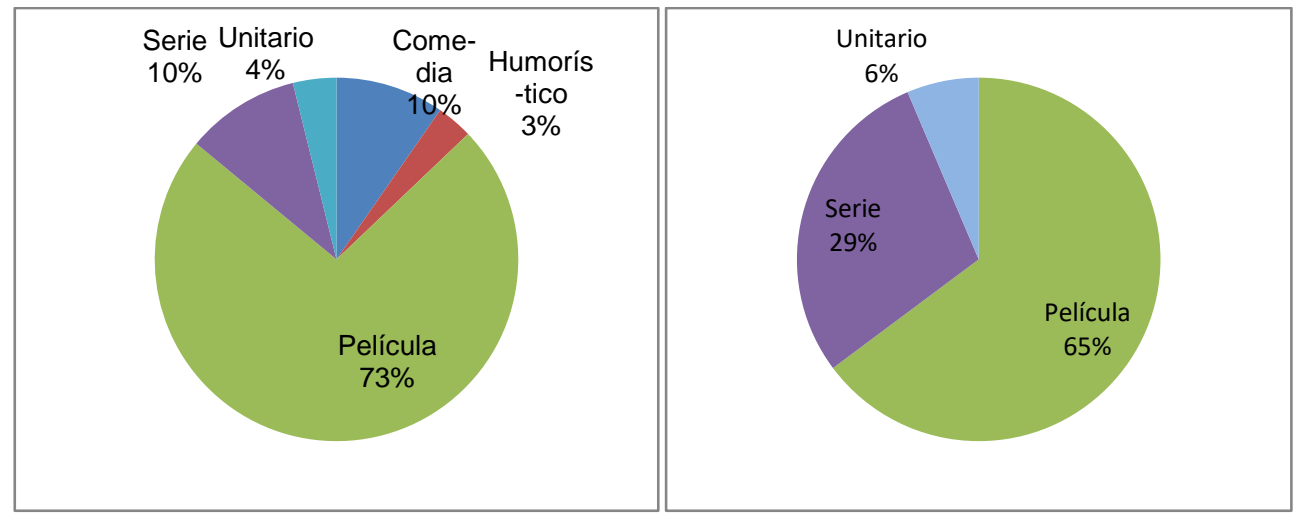

Marzo, del 28/03/16 al 03/04/16

Gráficos de elaboración propia

Abril, del 25/04/16 al 01/05/2016

Porcentaje de tiempo de emisión por género del discurso ficcional

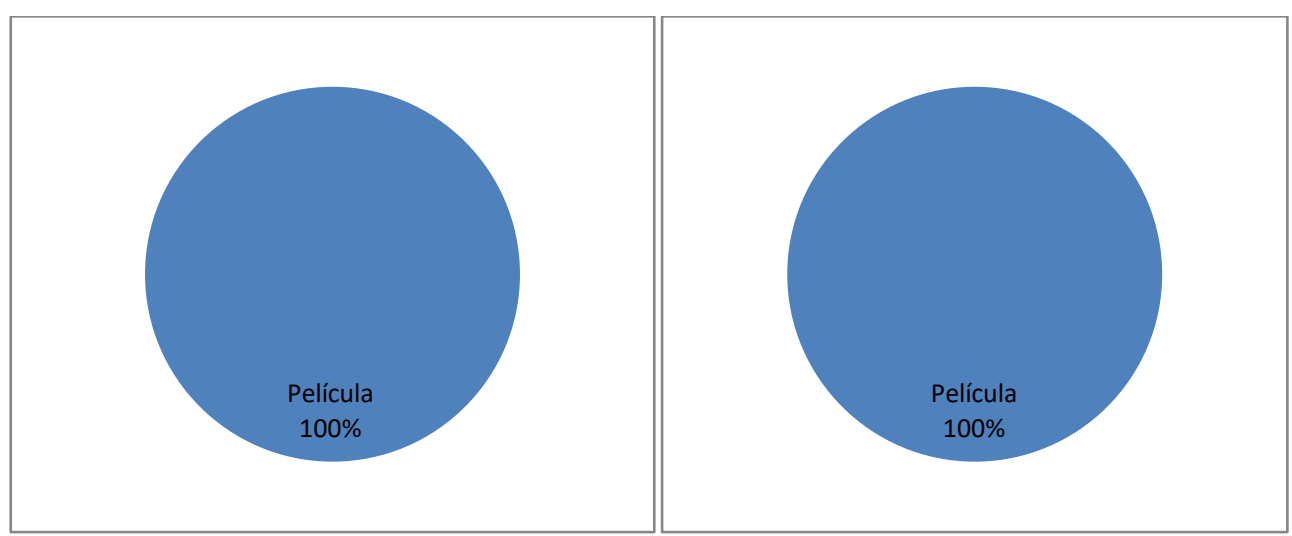

Marzo, del 23/03/19 al 30/03/19

Gráficos de elaboración propia

Si bien el género Película era el predominante en 2016, también se emitían otros géneros que en marzo y abril de 2019 dejaron de tener horas de pantalla. Entre otras cuestiones esto se corresponde con una baja de inversión en la ficción, de hecho varias de las series estrenadas durante 2016 -Ultimatum, El marginal, Mis noches sin ti, entre otras- fueron ganadoras de concursos para la promoción de la ficción del INCAA durante la gestión anterior ${ }^{12}$. Un dato inte-
Abril, del 20/04/19 al 27/04/19

resante lo aporta el informe elaborado por el Observatorio Ibero-americano de ficción televisiva:

El número de estrenos nacionales cae de 21 a 16 en relación a 2015 , debido a la retracción de la TV Pública, que estrena 7 títulos menos que el año anterior. Sin embargo, la cantidad de episodios y horas emitidas aumenta, ya que ganan peso los formatos de larga duración (Aprea, Kirchheimer y Rivero2017, p. 72).

La serie Cuéntame cómo pasó, estrenada en 2017 fue la única ficción producida íntegramente 
por la emisora. Esta tendencia de falta de inversión en la ficción se fue agudizando y en marzo y abril de 2019 sólo se emiten películas.

\subsection{PRIME TIME}

El horario de mayor encendido en la pantalla es durante la noche ${ }^{13}$, y por ende el más cotizado en términos publicitarios. Cada emisora ofrece su mejor apuesta en busca de mayor audiencia, aunque en el caso de la Televisión Pública, dado los números sobre la audiencia que ya hemos compartido, bien se podría cuestionar esta afirma- ción. Es cierto que en términos generales desde el 2006 el consumo de la televisión abierta ha ido disminuyendo sistemáticamente según los datos de Kantar IBOPE Media. La tendencia actual es hacia el crecimiento de la televisión on demand, siendo Netflix la empresa líder en servicio de streaming en América Latina (Baladrón y Rivero, 2017). Ahora bien, bajo este estado de situación, en 2019 la Televisión Púbica ofrece una programación en el horario de mayor encendido que se diferencia y distancia de las propuestas de los canales privados de la televisión abierta. Compartimos los siguientes gráficos que ilustran las propuestas, por género televisivo, en el horario del prime time.

Porcentaje de tiempo de emisión por géneros televisivos en horario del prime time

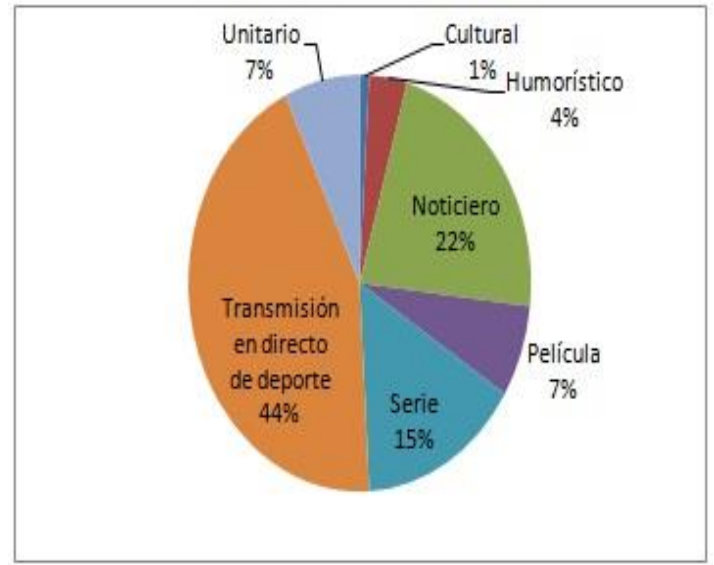

Marzo, del 28/03/16 al 03/04/16

Gráficos de elaboración propia

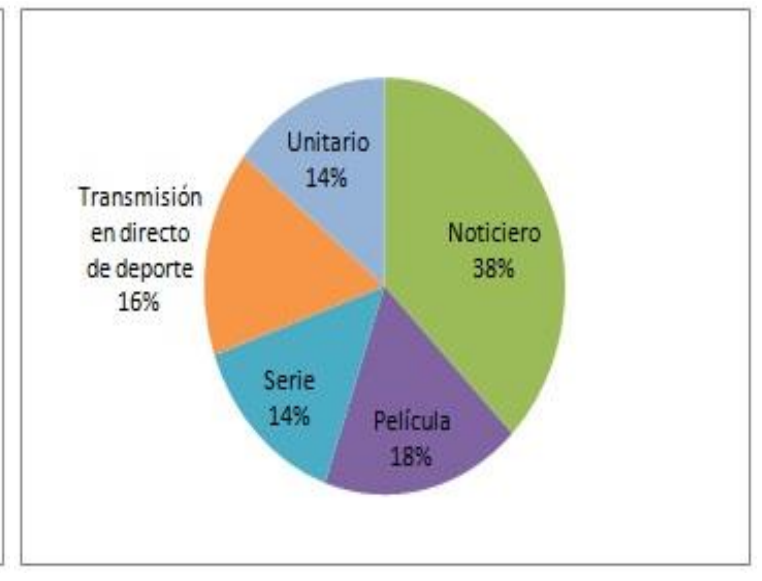

Abril, del 25/04/16 al 01/05/2016

Porcentaje de tiempo de emisión por géneros televisivos en horario del prime time

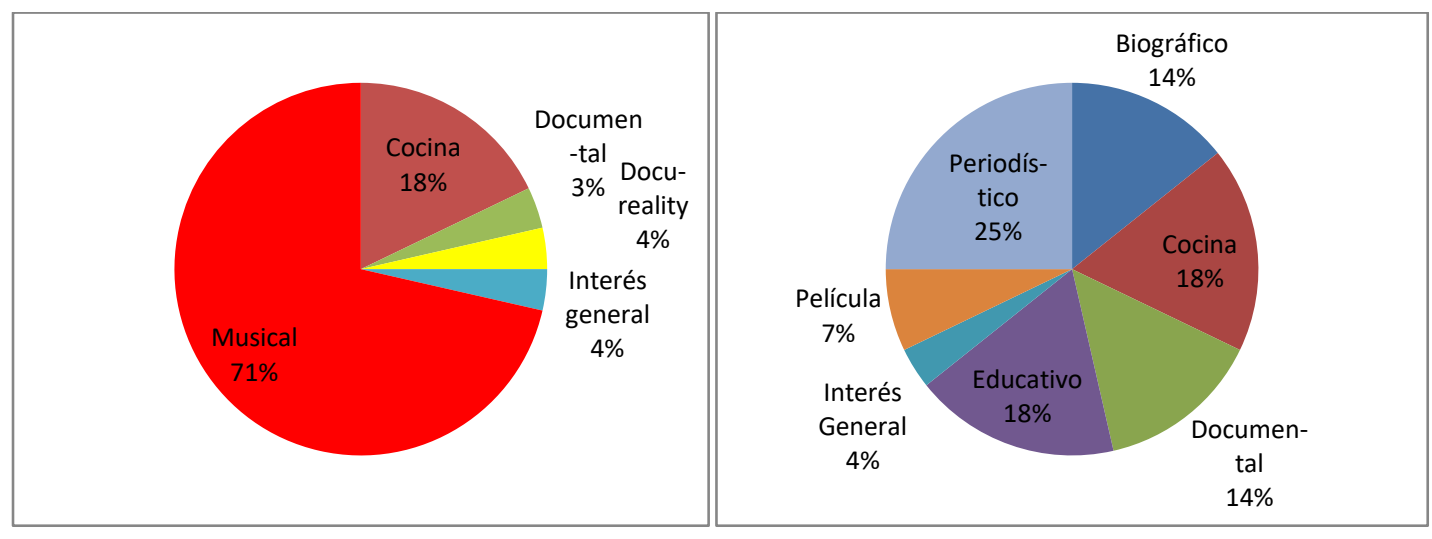

Marzo, del 23/03/19 al 30/03/19

Abril, del 20/04/19 al 27/04/19

Gráficos de elaboración propia 
Una primera cuestión a destacar es el cambio en la propuesta de la emisora. En 2016 además de la transmisión en vivo de deporte ${ }^{14}$ (se emitieron las eliminatorias para el mundial de fútbol Rusia 2018), durante el prime time se emitía ficción: series, unitarios y películas. En 2019 no se transmitieron contenidos ficcionales en el prime time, en sintonía con el descenso de este tipo de discurso en general en toda la programación. Como se mencionó anteriormente esto se debe a la falta de fomento a la ficción que trajo aparejado la disminución de producciones propias o coproducidas. Durante 2019 sólo se emitieron películas en la pantalla de la Televisión Pública y en horarios no centrales generalmente.

Otro cambio es que en 2019 el noticiero deja de emitirse en horario del prime time, pasando a las 19:00 horas. No es un dato menor si tenemos en cuenta los cambios llevados adelante por la gestión de Lombardi, entre otras cuestiones, la emisora dejó de emitir contenidos en vivo durante el fin de semana según el Informe de Reporteros sin Fronteras (2019), además del congelamiento de los salarios de los trabajadores y los despedidos. Según Linares y Mallimaci (2019):

Esto significó la eliminación de las horas extras y la reducción de hecho de los sueldos, que en algunos casos llega al 40\% del salario". Esto afectó directamente a los noticieros, según Linares y Mallimaci (2019) "esta política de reducción de costos disminuyó también la salida de móviles para transmisiones desde exteriores así como las coberturas periodísticas con enviados a acontecimientos internacionales (p. 12).

Otra cuestión a mencionar es que en marzo de 2019 ocupa un lugar destacado en el horario del prime time el género Musical, específicamente se emiten en directo Festivales, modificando la programación regular. Monje, Zanotti y Rivero (2017), afirman que "entre enero y marzo de 2017 la TV Pública transmitió 33 festivales desde diferentes ciudades del país (contra 15 transmisiones del año previo). Fueron en total 75 días y 400 horas de transmisión en horario central" ( $p, 167)$. Un rasgo diferenciador es que en el horario de mayor encendido hay una apuesta de la emisora por federalizar la transmisión vía festivales y fiestas populares. En un trabajo previo (Heram, 2018) en torno a la programación de 2016 en la Televisión Pública afirmábamos que "las propuestas de contenidos diferenciadoras se emiten en horarios periféricos, en las que el encendido es aún menor". Por el contrario en 2019 observamos que en el horario del prime time se emiten documentales, programas educativos, biográficos, de cocina, entre otros, aunque con niveles de audiencias muy bajos.

\subsection{PAÍS DE ORIGEN}

Uno de los temas que se discute cuando se reflexiona sobre el carácter y/o deber ser de los medios públicos es la necesidad de la inclusión de contenidos locales y regionales (Martín Barbero, 2005; Fuenzalida, 1998). Ahora bien, la inclusión de la cultura popular latinoamericana en términos de Fuenzalida no necesariamente se ve representada por el país de origen del producto, pero al menos nos permite visualizar de qué manera se piensa la programación. Compartimos los siguientes gráficos sobre el país de origen de los productos emitidos.

Porcentaje de tiempo de emisión por país de origen de los productos

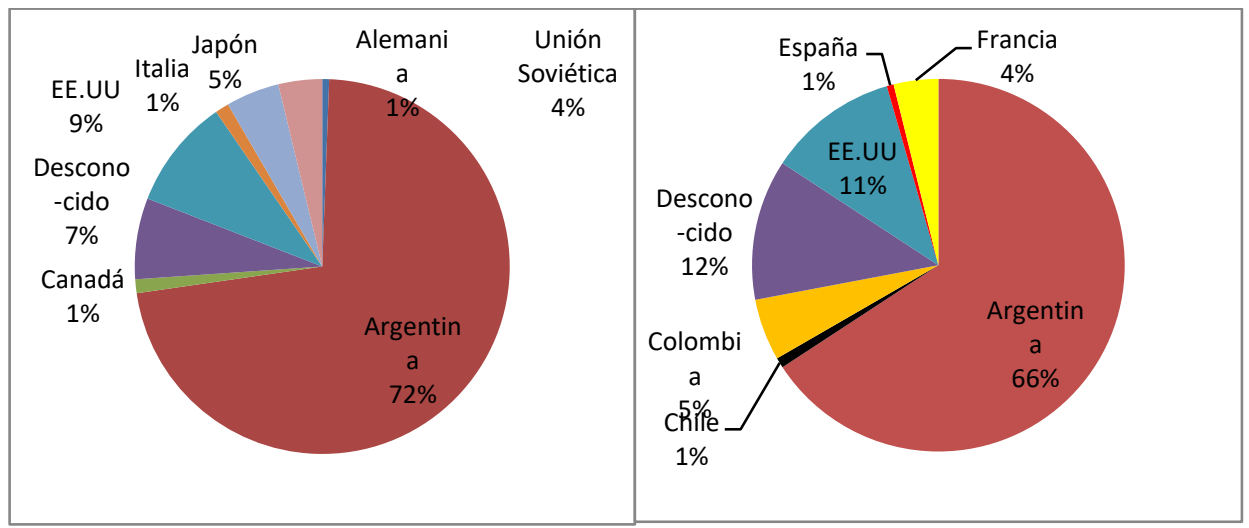

Marzo, del 28/03/16 al 03/04/16

Abril, del 25/04/16 al 01/05/2016

Gráficos de elaboración propia 


\section{ARTÍCULOS}

Porcentaje de tiempo de emisión por país de origen de los productos

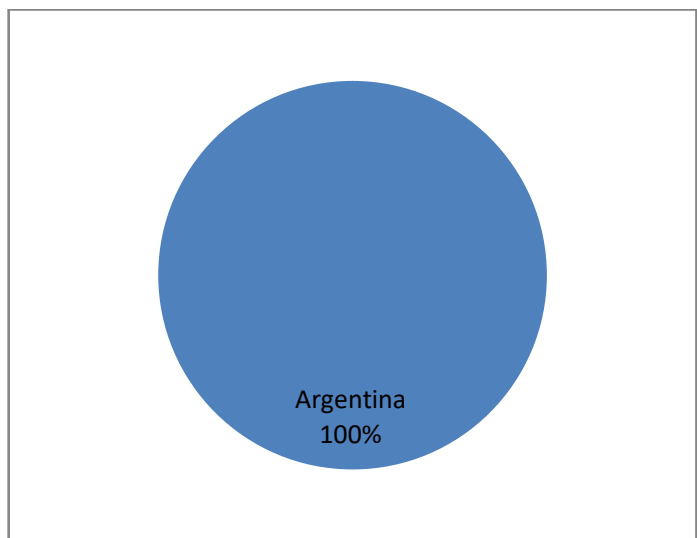

Marzo, del 23/03/19 al 30/03/19

Gráficos de elaboración propia 
En primer lugar cabe aclarar que si bien en términos porcentuales en los gráficos de 2019 figura $100 \%$ hubo unas pocas horas de emisión de productos de otros países, que al ocupar un porcentaje ínfimo, no fueron incorporadas en términos porcentuales porque no llegan al $1 \%$. Algunos días se emitieron películas de 90 minutos de duración, en el horario de la madrugada, provenientes de Reino Unido, Japón, Polonia y Estados Unidos.

En 2016 los productos provenientes de otros países se correspondían con la ficción, en su mayoría Películas y algunas Miniseries. En 2019, en consonancia con la disminución en general de horas de pantalla de la ficción, también se vio reducida a los contenidos extranjeros. En comparación con 2016 , la principal diferencia se ubica en la ausencia casi completa de productos provenientes de otros países. En 2019 encontramos una fuerte tensión entre el altísimo porcentaje de contenidos de origen local y la reducción en términos de inversión ${ }^{15}$ en los medios públicos, desde la disminución de programas en vivo, al congelamiento de los salarios, la falta de producción de ficción, entre otros. Estamos así en una suerte de paradoja, el momento en que más se emite producciones locales coincide con la disminución en términos de producción e inversión en los medios públicos locales.

\section{CONCLUSIONES}

A lo largo de este trabajo fuimos trazando algunas de las características de la programación de la Televisión Pública, para ello recurrimos a una amplia literatura proveniente de la economía política de la comunicación que permitió contextualizar y comprender el estado actual de la emisora, para evitar así un análisis aislado y descontextualizado de su programación. Una palabra se reiteró a lo largo del artículo - tensión - y creemos que sintetiza parte de nuestras conclusiones, hacemos referencia a: géneros referenciales diversos en el horario del prime time y un nivel de audiencia de los más bajos; mayor audiencia durante la gestión anterior y una pluralidad informativa cuestionada; mayor emisión de productos locales y disminución de inversión en los medios públicos. Además, varias de las decisiones tomadas en torno a la grilla de programación -desplazamiento y reducción de ficción en el horario central, inclusión de Festivales regionales, cambio en el horario del noticiero, entre otros- conlleva a una suerte de tensión mayor.
Por un lado, una propuesta de contenidos que difícilmente se encuentre en las señales privadas de aire y en especial en el horario de mayor encendido, la emisora funcionaría así como una suerte de complemento de aquello que los privados no ofrecen.

Por otra parte, nos preguntamos en un contexto como el actual donde el consumo se desplaza a los contenidos on demand, en el que las múltiples pantallas prevalecen como dispositivo de consumo, de qué manera la Televisión Pública, en su propuesta de cara a la ciudadanía, podría ofrecer otro tipo de formatos y contenidos que se adapten a los tiempos del streaming. Por solo poner un ejemplo, se siguen emitiendo películas clásicas de la década de 1940, ¿esto se debe a que la emisora busca llegar a otros rangos etarios alejados de los consumos on demand? ¿Lo hace para ofrecer una propuesta diferenciadora? ¿Podría ser un film contemporáneo también otra manera de diferenciarse y competir? Estas preguntas son solo algunas que cristalizan la complejidad de pensar en una programación diferente y competitiva, y que a su vez se caracterice por las cuestiones centrales que los medios públicos deberían brindar a la ciudadanía - pluralismo, calidad, diversidad, experimentación entre otros aspectos. Nos preguntamos entonces, sin caer en el binarismo dicotómico de contenidos culturales o mayor audiencia, no solo de qué manera se podría contemplar la cuestión de los espectadores en los medios públicos, sino qué tipo de contenidos y para quién.

Por último y para cerrar, si algo ha caracterizado a las emisoras públicas de América Latina ha sido la permanente tensión entre características de los medios públicos, estatales y gubernamentales. Una amplia literatura - mucha de ella citada - ha discutido en torno a esto. Creemos que los resultados de esta investigación si bien focalizan en el caso de Argentina son un insumo relevante para pensar las televisoras públicas en Latinoamérica, pudiendo así en un futuro realizar, a partir de la metodología propuesta, análisis comparativos que permitan ampliar la discusión sobre el devenir de los medios públicos. 


\section{NOTAS}

1 Cfr. de Charras, 2016; Mastrini, 2013; Mastrini y Becerra, 2017; Rincón, 2005; Cullel, 2010; Guérin, Miranda, Oliveri, Santagata, 2013; Arroyo, Becerra, García Castillejo, Santamaría, 2012; Fuenzalida 1998, 2002; Becerra 2015 Ortega, P. 2010; Linares 2018; Monje, Zanotti y Rivero 2017: entre otros.

2 Los medios sin fines de lucro, que menciona la Ley de Servicios de Comunicación Audiovisual, no fueron implementados o lo hicieron de manera parcial (Mastrini y Becerra, 2017; Marino, 2016; Guimerà Orts y Marino, 2016; Segura y Weckesser, 2016; Segura, 2013).

3 Ello nos obliga a pensar en otros términos si lo comparamos con los medios públicos europeos que se expandieron después de la Segunda Guerra Mundial, ya que como menciona Germán Rey "la naturaleza de lo público ha ido cambiando" (2005, p. 70)

4 Proyecto de investigación PICT (2020 - 2022) sobre la televisión contemporánea, dirigido por la autora.

5 En abril de 2016 se lanzó oficialmente la nueva programación.

6 Para mayor información:

https://www.boletinoficial.gob.ar/\#!DetalleNorma/139608/ 20160105

7 Sobre este tema se puede ampliar con Marino (2016); Becerra (2016)

8 Un fallo judicial a favor de los empleados obliga a su reincorporación.

9 Primer canal público, cultural y educativo creado por el Ministerio de Educación en 2005. Para mayor información se puede consultar Smerling (2015).

$10 \mathrm{Al}$ respecto hay una serie de discusiones en torno a si un programa como 678 debía transmitirse la señal pública debido a que los medios comerciales representaban a otros grupos e intereses (Bonavetti, 2013; Alabarces y Oliván, 2010).

11 Los trabajadores del noticiero emiten \#NotitrabajadoresTVP, un noticiero donde denuncian las políticas de ajuste y vaciamiento a los medios públicos por parta de Hernán Lombardi.

12 Para mayor ampliación algunos artículos de Página/12 hacen eco de la situación (Respighi, 7 de junio de 2016; 29 de marzo de 2016; 13 de abril de 2016).

13 Si bien puede variar en cada país, en Argentina abarca desde las 20:00 a las 24:00 horas.

14 Se privatizó la transmisión de Fútbol de Primera División en 2016.

15 Cfr. Linares y Mallimaci (2019).

\section{REFERENCIAS BIBLIOGRÁFICAS}

- Alabarces, P. y Oliván, M. J. (2010). 67 8. La creación de la otra realidad. Paidós.

- Arroyo, L.; Becerra, M.; García Castillejo, A. \& Santamaría, O. (2012). Cajas mágicas. EI renacimiento de la televisión pública en América Latina. Tecnos.

- Aprea, G., Kirchheimer. M., y Rivero, E. (2017). Argentina: cambio de rumbo en la producción nacional. Concentración y refuerzo de las lógicas comerciales. En Vassallo de Lopes, M. I. y Orozco Gómez, G. Una década de ficción televisiva en Iberoamérica. Análisis de 10 años de Obitel (pp. 63-92). Globo Comunicação e Participações.

- Baladrón, M. y Rivero, E. (2017). La regulación de las plataformas OTT audiovisuales: un modelo para armar. Fibra. Tecnologías de la Comunicación, (16) 1-6. Recuperado de:

http://papel.revistafibra.info/la-regulacion-lasplataformas-ott-audiovisuales-modelo-armar/

- Becerra, M. (2015). De la concentración a la convergencia. Políticas de medios en Argentina y América Latina. Paidós.

- Becerra, M. (2013). La televisión pública en América Latina: condicionantes y desafíos. En Guérin, A. I., Miranda A., Olivieri, R., Santagata, G. (comps.), Televisión Pública ¿Qué modelos para América Latina?(pp. 29-53). La Crujía.

- Becerra, M. (2016). Hechizo de tiempo en los medios estatales. Colsecor Revista, (256). Recuperado de:

https://martinbecerra.wordpress.com/2016/12/1 3/hechizo-de-tiempo-en-los-medios-estatales/

- Bonavetti, M. (2013). Identidad, pluralismo y diversidad. Televisión Pública para el siglo XXI. En Guérin, A. I., Miranda A., Olivieri, R., Santagata, G. (comps). Televisión Pública ¿Qué modelos para América Latina?(pp. 355-366). La Crujía.

- Cullel, R. M. (2010). La televisión pública frente al desafío digital ¿Mantener la calidad o la audiencia? TELOS (84), 102-104.

- de Charras, D. (comp.) (2016). Implementación del sistema de indicadores de calidad de emisoras públicas para la evaluación de la Televisión Pública Argentina. Universidad de Buenos Aires. Facultad de Ciencias Sociales.

- Denzin, N. \& Lincoln, Y. (2003). Strategies of qualitative inquiry. Sage y Thousand Oaks.

- Fuenzalida, V. (1998). Situación de la TV-Pública en América Latina. Diálogos de la Comunicación, (53).

- Fuenzalida, V. (2002). Televisión abierta y audiencia en América Latina. Norma.

- Guérin, A. I., Miranda A., Olivieri, R., Santagata, G. (comps). Televisión Pública ¿Qué modelos para América Latina? La Crujía.

- Guimerà Orts, J. A. y Marino, S. (2016). Televisión sin fines de lucro en la Argentina de la Ley Audiovisual: el caso de Barricada TV. Austral 
Comunicación, 5(2), 205-227. DOI:

https://doi.org/10.26422/aucom.2016.0502.mar

- Heram, Y. (2018). Televisión Pública: un estudio exploratorio de su programación. Estudos em Comunicação, 1(26), 65-80. Recuperado de: http://ojs.labcomifp.ubi.pt/index.php/ec/article/view/202

- Heram, Y., Toledo, C., Merchert, M. Y Bleiz, C. (2017). Tendencias actuales de los canales de aire de la televisión argentina. Ciencias Sociales (94), 138-141.

- Linares, A. (2018). El financiamiento en los medios estatales de Argentina entre 2003 y 2015 . Cambios y continuidades dentro de una tendencia de expansión. Commons, (7), 95-132. DOI: http://doi.org/10.25267/COMMONS.2018.v7.i2

- Linares, A. y Mallimaci, M. A. (2019). Los medios estatales de Argentina a partir de la asunción de la alianza Cambiemos en el gobierno nacional (2016 2018). Question, 1(61), 1-18.

- Marino, S. (2016). La nueva política comunicacional: consecuencias mediáticas. Anfibia, 1-8. Recuperado de:

http://revistaanfibia.com/ensayo/consecuenciasmediaticas /

- Martín-Barbero, J. (1987). De los medios a las mediaciones. Gustavo Gilli.

- Martín-Barbero, J. (2005). Claves de debate: televisión pública, televisión cultural: entre la renovación y la invención. En Rincón, O. (ed.) (2005). Televisión pública: del consumidor al ciudadano (pp. 35-68). La Crujía.

- Mastrini, G. (2013). Medios públicos y derecho a la comunicación. En Mastrini, G. Bizberge, A. y de Charras, D. (eds.), Las políticas de comunicación en el siglo XXI. Nuevos y viejos desafíos. La Crujía.

- Mastrini, G. y Becerra, M. (2017). Medios en guerra: balance, crítica y desguace de las políticas de comunicación 2003-2016. Biblos.

- Mindez, L. (2001). Canal Siete. Medio siglo perdido. La historia del Estado argentino y su estación de televisión. La Crujía.

- Monje, D., Zanotti, M. y Rivero, E. A. (2017). Contrarreforma en la TV Pública Argentina: cambios regresivos y reinvención cíclica. Eptic, 19(3), 1-19. Recuperado de: https://seer.ufs.br/index.php/eptic/article/view/ 7225

- Ortega, P. (2010). Televisión Pública en América Latina. Los valores del mercado y las políticas del Estado. Infoamérica ICR, 205-213.

- Orza, G. (2002). Programación televisiva. Un modelo de análisis instrumental. La Crujía.

- Reporteros sin Fronteras. (2019) ¿Quiénes son los dueños de los medios en Argentina? Recuperado de: http://argentina.mom-rsf.org/es/

- Respigui, E. (2016, 7 de junio). A nuestros personajes los une el sentido de humor. Página/12. Recuperado de:

https://www.pagina12.com.ar/diario/suplemento s/espectaculos/8-39084-2016-06-07.html

- Respigui, E. (2016, 29 de marzo). Una pantalla a puro magazine. Página/12. Recuperado de: https://www.pagina12.com.ar/diario/suplemento s/espectaculos/8-38390-2016-03-29.html

- Respigui, E. (2016, 13 de abril). Por ahora, magazine y entretenimiento. Página/12. Recuperado de:

https://www.pagina12.com.ar/diario/suplemento s/espectaculos/8-38547-2016-04-13.html

- Rey Beltrán, G. (2005). Panorama: el escenario móvil de la televisión pública. Algunos elementos del contexto. En Rincón, O. (ed.). Televisión pública: del consumidor al ciudadano (pp. 69-012). La Crujía.

- Ricaurte, C. (eds.) (2009). La palabra rota. Seis investigaciones sobre el periodismo ecuatoriano. Fundamedios.

- Rincón, O. (ed.) (2005). Televisión pública: del consumidor al ciudadano. La Crujía.

- S/A. (2019). Boletín oficial de la República Argentina. Buenos Aires. Recuperado de: https://www.boletinoficial.gob.ar/\#!DetalleNorma /139608/20160105

- S/A. (2019). KANTAR IBOPE MEDIA. Asunción. Recuperado de: https://www.kantaribopemedia.com/kttr-typeargentina/

- Salviolo, C. (2013). Pakapaka: la construcción de un nuevo relato sobre la infancia. En Guérin, A. I., Miranda A., Olivieri, R., Santagata, G. (comps). Televisión Pública ¿Qué modelos para América Latina? (pp. 409-420). La Crujía.

- Sánchez-Carrero, J. y Méndiz-Rojas, H. (2013). La alfabetización mediática en la televisión infantil online: programas del Canal Pakapaka. Chasqui, (124), 55-62. Recuperado de: http://hdl.handle.net/10272/7636

- Segura, M. S. (2013). Contigo o sin ti: medios no lucrativos y Estado desde la Ley 26.522. Austral 
comunicación, 2(2), 145-185. DOI:

https://doi.org/10.26422/aucom.2013.0202.gon

- Segura, M. S. y Weckesser, C. (2016). Los medios sin fines de lucro entre la Ley Audiovisual y los decretos: estrategias, desafíos y debates en el escenario 2009- 2015. Editorial de la Universidad Nacional de Córdoba.

- Smerling, T. (2018, 27 de septiembre). Pakapaka y el peligro de la imaginación. Letra $P$. Recuperado de: https://www.letrap.com.ar/nota/2018-9-2710-28-0-pakapaka-y-el-peligro-de-la-imaginacion

- Smerling, T. (2015). La otra pantalla: educación, cultura y televisión. 2005 -2015, una década de Canal Encuentro, Paka Paka y las nuevas señales educativas. Buenos Aires: edicioneseduc.ar. 